This is the abstract and link to the article in the Wiley journal - Journal of Computer Assisted Learning (JCAL) - published December 2018.

Free read-only access to full article: https://rdcu.be/401M

\title{
Virtual Reality and Situated Experiential Education: A conceptualisation and exploratory trial
}

Christian Schott and Stephen Marshall

Victoria University of Wellington

P.O. Box 600

Wellington

New Zealand

Corresponding author: christian.schott@vuw.ac.nz

\begin{abstract}
Virtual reality is widely recognised as offering the potential for fully immersive environments.

This paper describes a framework that guides the creation and analysis of immersive environments that are pedagogically structured to support situated and experiential education. The "situated experiential education environment" framework described in this paper is used to examine the impact that a virtual environment can have on the user experience of participants in a virtual space. The analysis of a virtual environment implemented to support learner exploration of issues of tourism development and the related impacts suggests that this type of experience is capable of providing participants with a holistic experience of real world environments that are otherwise too expensive, impractical or unethical for large groups of people to visit in person. The pedagogical value of such experiences is enabled through immersion in a reality-based environment, engagement with complex and ambiguous situations and information, and interaction with the space, other students and teachers. The results
\end{abstract}


demonstrate that complex immersive learning environments are readily achievable but that high levels of interactivity remains a challenge.

Keywords: experiential education, situated cognition, digital immersion, virtual reality

Free read-only access to full article: https://rdcu.be/401M 